\title{
Functional Magnetic Resonance Imaging Investigation of Overlapping Lateral Occipitotemporal Activations Using Multi-Voxel Pattern Analysis
}

\author{
Paul E. Downing, Alison J. Wiggett, and Marius V. Peelen \\ School of Psychology, University of Wales, Bangor, Gwynedd LL57 2AS, United Kingdom
}

\begin{abstract}
Several functional areas are proposed to reside in human lateral occipitotemporal cortex, including the motion-selective human homolog of macaque area MT (hMT), object-form-selective lateral occipital complex (LO), and body-selective extrastriate body area (EBA). Indeed, several functional magnetic resonance imaging (fMRI) studies have reported significant activation overlap among these regions. The standard interpretation of this overlap would be that the common areas of activation reflect engagement of common neural systems. Alternatively, motion, object form, and body form may be processed independently within this general region. To distinguish these possibilities, we first analyzed the lateral occipitotemporal responses to motion, objects, bodies, and body parts with whole-brain group-average analyses and within-subjects functional region of interest (ROI) analyses. The activations elicited by these stimuli, each relative to a matched control, overlapped substantially in the group analysis. When hMT, LO, and EBA were defined functionally within subjects, each ROI in each hemisphere (except right-hemisphere hMT) showed significant selectivity for motion, intact objects, bodies, and body parts, although only the peak voxel of each region was tested. In contrast, multi-voxel analyses of variations in selectivity patterns revealed that visual motion, object form, and the form of the human body elicited three relatively independent patterns of fMRI activity in lateral occipitotemporal cortex. Multi-voxel approaches, in contrast to other methods, can reveal the functional significance of overlapping fMRI activity in extrastriate cortex and, by extension, elsewhere in the brain.
\end{abstract}

Key words: fMRI; motion; object recognition; occipital; temporal; visual selectivity

\section{Introduction}

Evidence from functional magnetic resonance imaging (fMRI) studies indicates that at least three functional areas occupy similar territory in the human lateral occipitotemporal cortex (LOTC). These include the following: (1) a proposed human homolog of macaque area MT (hMT) (Tootell et al., 1995), a region that responds selectively to visual motion; (2) the dorsal focus of the lateral occipital complex (LO) (Malach et al., 1995; Grill-Spector et al., 1999), a region that responds selectively to familiar or unfamiliar objects compared with scrambled controls; and (3) the extrastriate body area (EBA) (Downing et al., 2001), a region that responds selectively to human bodies and body parts compared with objects and other control stimuli. Indeed, several studies have reported substantial overlap between these areas (Downing et al., 2001; Kourtzi et al., 2002; Spiridon et al., 2005; Peelen et al., 2006). How should this overlap be interpreted?

The standard interpretation would be that the overlapping region contains neurons engaged in a common computational process shared by the experimental conditions (but not their respective controls). For example, this interpretation has been the

Received Aug. 21, 2006; revised Nov. 28, 2006; accepted Nov. 30, 2006.

This work was supported by the Biotechnology and Biological Sciences Research Council.

Correspondence should be addressed to Dr. Paul E. Downing, Centre for Cognitive Neuroscience, School of Psy-

chology, University of Wales, Bangor, Gwynedd LL57 2AS, UK. E-mail: p.downing@bangor.ac.uk.

DOI:10.1523/JNEUROSCI.3619-06.2007

Copyright $\odot 2007$ Society for Neuroscience $\quad$ 0270-6474/07/270226-08\$15.00/0 favored account of prefrontal brain areas activated by both observed and performed manual actions and, in this case, has been taken as evidence for a "mirror neuron" system in the human brain (Iacoboni et al., 1999). fMRI studies of phonological retrieval (Price and Friston, 1997), multiple-task performance (Jiang and Kanwisher, 2003), empathy (Singer et al., 2004), and visual attention (Wojciulik and Kanwisher, 1999; Marois et al., 2004) have followed similar logic to make claims about cognitive/ neural mechanisms shared across multiple tasks or stimuli. However, a plausible alternative account of the overlap between hMT, LO, and EBA (and perhaps also for overlapping activations in other brain areas) is that commonly activated voxels contain independent neural systems, each coding for a stimulus or task dimension but intermixed at a resolution below that of individual fMRI voxels.

How can these accounts be distinguished? Recent developments in fMRI data analysis reveal that substantially more information is present in the response of a brain region than is captured by its overall mean response to various experimental conditions (Haxby et al., 2001; Haynes and Rees 2006; Norman et al., 2006). Analysis of the local patterns of neural activity on a voxelwise basis can detect small but stable variations in selectivity. These can be used, for example, to "read" neural activity to distinguish perceptual states (Haynes and Rees, 2005; Kamitani and Tong, 2005). We have used similar approaches in recent work to disentangle the sources of responses to biological motion 


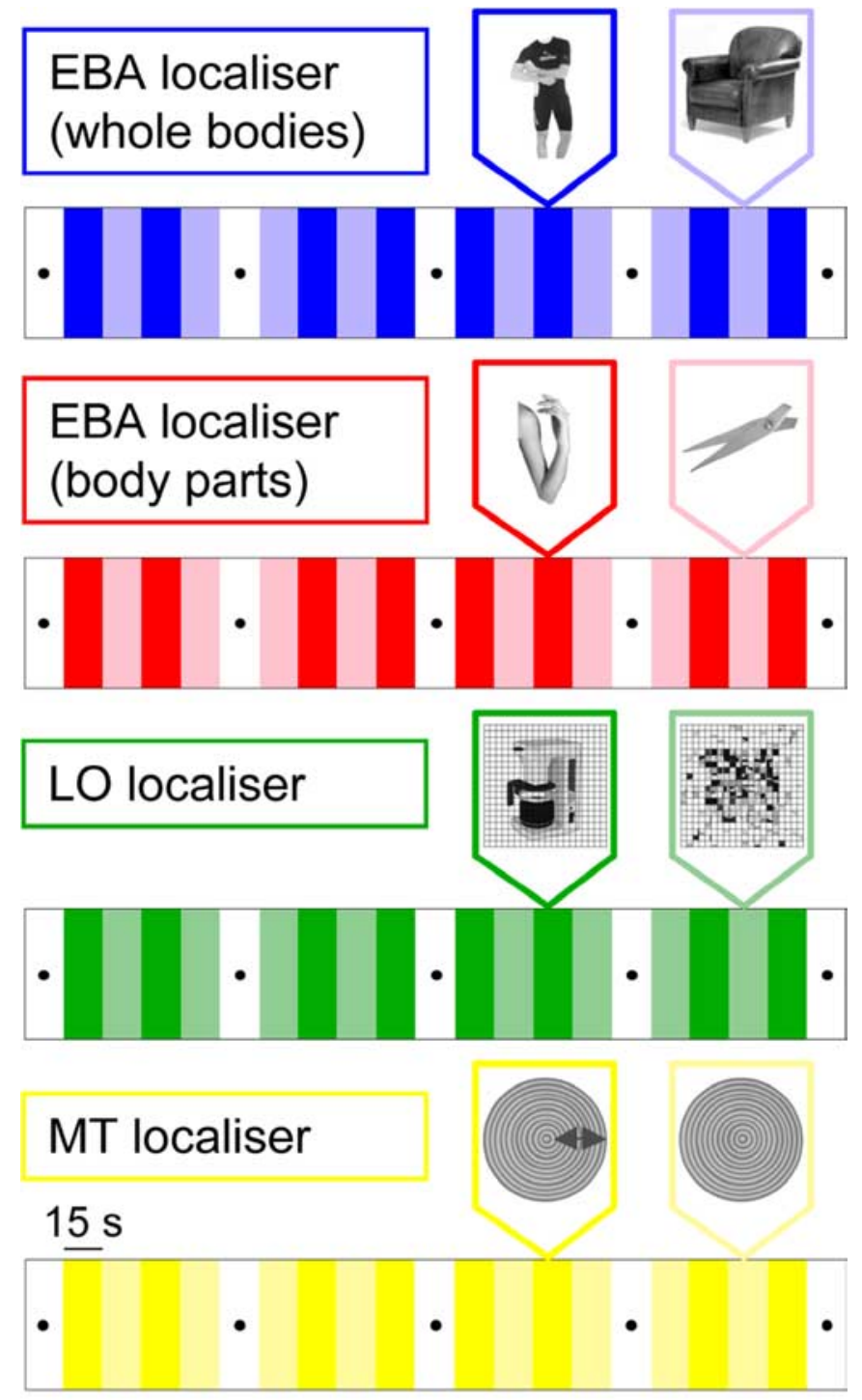

time

Figure 1. Schematic of experimental designs.

in overlapping functional regions of interest (fROIs) (Peelen et al., 2006).

Here we applied this technique to the analysis of overlapping activations in the LOTC. The main approach of this study was as follows. First, we analyzed the fMRI responses to motion, objects, bodies, and body parts with standard whole-brain group-average analyses and within-subjects fROI analyses. We then compared those results with multi-voxel analyses of local variations in selectivity patterns in the same area, to determine whether these patterns were more sensitive to the underlying neural organization than standard analyses.

\section{Materials and Methods}

Subjects. Sixteen healthy adult volunteers were recruited from the University of Wales, Bangor community. Participants satisfied all requirements in volunteer screening and gave informed consent approved by the School of Psychology at the University of Wales, Bangor. Participation was compensated at $£ 20$ per session.

Design and procedure. Each participant was scanned on four blockeddesign fMRI experiments (Fig. 1): two EBA localizers (using whole bodies or body parts), one LO localizer, and one hMT localizer. Each exper- iment served two purposes: to identify fROIs and to test the response selectivities in all other ROIs (e.g., motion selectivity in EBA).

We localized EBA with two different experiments: one consisting of blocks of images of human bodies (without heads) and images of chairs, which we label "wEBA"; and one consisting of blocks of images of body parts and object parts, which we label "pEBA." For the LO localizer scan, we tested images of objects and scrambled versions of the same images. The basic design of the EBA (whole and parts) and LO experiments was identical: each experiment consisted of $2115 \mathrm{~s}$ blocks in which blocks 1 , $6,11,16$, and 21 were fixation-only baseline epochs. In each of the remaining blocks, 20 different images from one category (wEBA, bodies or chairs; pEBA, body parts or object parts; LO, intact or scrambled objects) were presented. Each image was presented for $300 \mathrm{~ms}$, followed by a blank screen for $450 \mathrm{~ms}$. Twice during each stimulus block, the same image was presented two times in succession. Participants were instructed to detect these immediate repetitions and report them with a button press (1-back task). The position of the image was jittered slightly on alternate presentations, to disrupt attempts to perform the 1-back task based on low-level visual transients. Each participant was tested with two different versions of each experiment, counterbalancing for the order of stimulus category. In both versions, assignment of category to block was counterbalanced, so that the mean serial position in the scan of each condition was equated.

The blocked-design localizer for area hMT consisted of a pattern of low-contrast, concentric rings that either slowly oscillated inward or outward, or, in separate blocks, remained static (cf. Tootell et al., 1995). The experiment again consisted of 2115 s blocks; blocks 1, 6, 11, 16, and 21 were fixation-only epochs. In the remaining blocks, moving and static conditions were alternated. The stimuli in this experiment were passively viewed. Participants were tested with two runs of this experiment. In total, each session consisted of eight scans.

Data acquisition. A 1.5T Philips MRI scanner with a SENSE parallel head coil was used. For functional imaging, a single-shot echo planar imaging sequence was used ( $\mathrm{T} 2^{\star}$-weighted, gradient echo sequence; echo time, $50 \mathrm{~ms}$; flip angle, $90^{\circ}$ ). The scanning parameters were as follows: repetition time, $2000 \mathrm{~ms}$; 20 off-axial slices; voxel dimensions, $3 \times 3 \mathrm{~mm}$; $4 \mathrm{~mm}$ slice thickness.

Preprocessing. Preprocessing and statistical analysis of MRI data were performed using BrainVoyager 4.9.6 (Brain Innovation, Maastricht, The Netherlands). Three dummy volumes were acquired before each scan to reduce possible effects of $\mathrm{T} 1$ saturation. Functional data were motion corrected, and low-frequency drifts were removed with a temporal highpass filter $(0.006 \mathrm{~Hz})$. No spatial smoothing was applied. Functional data were manually coregistered with three-dimensional anatomical $\mathrm{T} 1 \mathrm{scans}$ $(1 \times 1 \times 1.3 \mathrm{~mm}$ resolution). The three-dimensional anatomical scans were transformed into Talairach space (Talairach and Tournoux, 1988), and the parameters from this transformation were subsequently applied to the coregistered functional data.

Whole-brain analysis. Whole-brain random-effects, group-average analyses were conducted on data from all four experiments. Contrasts were performed at uncorrected thresholds of $p<0.0005$ and $p<0.0001$ to test for regions significantly more active to (1) bodies than chairs, (2) body parts than object parts, (3) intact than scrambled objects, or (4) moving than static rings. Only clusters $>100 \mathrm{~mm}^{3}$ are reported for these analyses.

ROI analyses. For each participant, general linear models (GLMs) were created for each experiment. One predictor, convolved with a delayed gamma function $(\delta=2.5 \mathrm{~s}$; tau $=1.25 \mathrm{~s})$ to model the hemodynamic response (Boynton et al., 1996), modeled each condition of interest. Regressors of no interest were also included to account for differences in the mean MR signal across scans. Regressors were fitted to the MR time series in each voxel, and the resulting $\beta$ parameter estimates were used to estimate the magnitude of response to each experimental condition.

For each ROI (hMT, LO, wEBA, pEBA) in each hemisphere of each subject, the most significantly activated voxel was identified within a restricted part of cortex based on previously reported anatomical locations [EBA (Peelen and Downing, 2005b), hMT (Dumoulin et al., 2000), LO (Kourtzi et al., 2003)]. Each ROI was defined solely by its "peak" voxel (i.e., the voxel with the highest $t$ value). 
Voxelwise correlation analyses. For each subject, we created an additional GLM that contained all runs from all four experiments. We used this GLM to contrast the response to the average of (human bodies, body parts, intact objects, and moving rings) versus the average of (chairs, object parts, scrambled objects, and static rings). This resulted in general left- and right-hemisphere LOTC activations for each subject that are responsive to bodies, body parts, motion, and intact objects but that have not been parceled into discrete functional areas. We identified the most significantly activated voxel of this activation in each subject and hemisphere and defined a "combined ROI" as all voxels around this peak that were significantly activated ( $p<0.00001$, uncorrected) by bodies, body parts, intact objects, and moving rings. We restricted the region to a $30 \times 30 \times 30$ $\mathrm{mm}$ cube around the peak voxel.

Two additional sets of ROIs were generated by first identifying an hMT, LO, and EBA ROI for each subject and hemisphere and then iden-

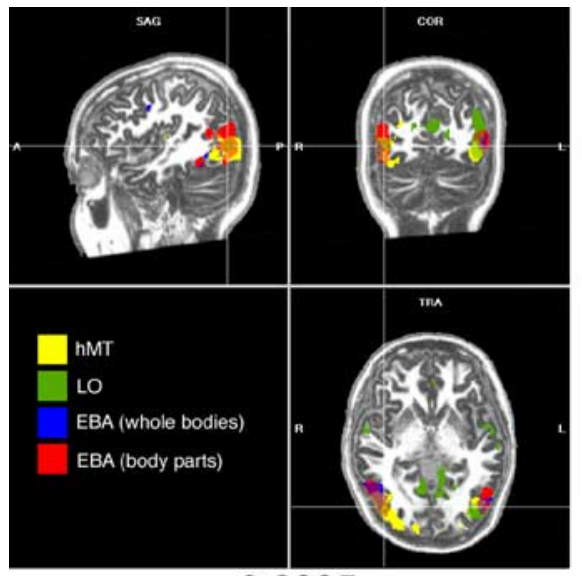

$\mathrm{p}<0.0005$

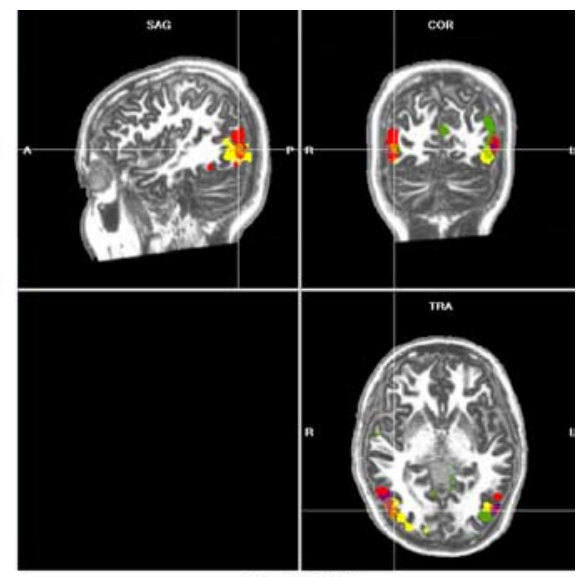

$\mathrm{p}<0.0001$

Figure 2. Results of random-effects group-average contrasts of moving versus static rings (hMT; yellow), intact versus scrambled objects ( $L 0$; green), human bodies versus chairs (EBA; red), and human body parts versus object parts (EBA; blue). Results are shown at $p<0.0005$ and $p<0.0001$, uncorrected. The slices shown are at Talairach coordinates $+42,-72,+1$. A, Anterior; $P$, posterior; R, right; L, left; SAG, sagittal; COR, coronal; TRA, transverse.

Table 1. Talairach and Tournoux (1988) coordinates of the peak voxel of each ROI, from the group-average, random-effects analysis

\begin{tabular}{|c|c|c|c|c|c|c|}
\hline & \multicolumn{3}{|l|}{ Left } & \multicolumn{3}{|c|}{ Right } \\
\hline & $x$ & $y$ & $z$ & $x$ & $y$ & $z$ \\
\hline EBA (bodies) & -51 & -68 & 3 & 51 & -67 & 3 \\
\hline EBA (body parts) & -48 & -67 & 6 & 48 & -70 & 3 \\
\hline hMT & -36 & -76 & -8 & 41 & -64 & -2 \\
\hline LO & -39 & -73 & 0 & 45 & -76 & 0 \\
\hline
\end{tabular}

Table 2. Mean Talairach and Tournoux (1988) coordinates of the peak voxel, across subjects, of each ROI

\begin{tabular}{|c|c|c|c|c|c|c|}
\hline & \multicolumn{3}{|l|}{ Left } & \multicolumn{3}{|l|}{ Right } \\
\hline & $x$ & $y$ & $z$ & $x$ & $y$ & $z$ \\
\hline EBA (bodies) & $-47(4)$ & $-72(6)$ & $1(6)$ & $51(4)$ & $-63(6)$ & $1(6)$ \\
\hline EBA (body parts) & $-46(6)$ & $-70(6)$ & $4(7)$ & $49(5)$ & $-67(6)$ & $3(5)$ \\
\hline hMT & $-44(5)$ & $-67(6)$ & $1(6)$ & $47(4)$ & $-65(5)$ & $0(5)$ \\
\hline LO & $-45(6)$ & $-70(7)$ & $-3(7)$ & $46(4)$ & $-71(6)$ & $-4(8)$ \\
\hline
\end{tabular}

SDs are given in parentheses. Note that the average peaks of these regions are highly similar.

tifying voxels on the basis of assignment to these regions (at an uncorrected threshold of $p<0.0001$ ). EBA was defined as the union of voxels in wEBA and pEBA. "Union ROIs" were then defined for each subject and hemisphere by including the voxels in the union of hMT, LO, and EBA. "Intersection ROIs" were also defined for each subject and hemisphere by selecting only voxels that were in the intersection of hMT, LO, and EBA. Union and intersection ROIs were restricted to a $50 \times 50 \times 50$ $\mathrm{mm}$ cube around the peak voxel.

For all three ROI types (combined, union, and intersection), in each subject and hemisphere, we measured the voxelwise pattern of selectivity to each key stimulus type (bodies, body parts, intact objects, and moving rings) compared with its associated control condition. This was done by extracting a $t$ value for a given contrast (e.g., bodies - chairs) at each voxel within the ROI (see also Peelen et al., 2006). This was done in each subject for each of the eight scans. A correlation between two selectivity patterns (e.g., body and motion selectivity) was calculated as follows. First, we computed a $t$ value for each voxel in the ROI reflecting body selectivity (by contrasting bodies vs chairs). Second, we computed a $t$ value for each voxel in the same ROI reflecting motion selectivity (by contrasting moving vs static rings). These two sets of $t$ values were then represented as two one-dimensional vectors. The correlation between these two vectors constitutes the voxelwise correlation between body and motion selectivity.

We selected the $t$ value as a measure of selectivity because it captures in one value the size and robustness of the differential response in a voxel to two experimental conditions. An additional advantage of characterizing selectivity for each voxel relative to a baseline [as opposed to a normalized absolute activation value (Kamitani and Tong, 2006)] is that lowlevel aspects of the response to visual stimulation per se are controlled for. This reduces the impact of variations between voxels in relative gray/ white matter content, which would be expected to produce uninformative positive correlations between response patterns.

In each ROI for each subject, we correlated the pattern of selectivity for one contrast with the pattern of selectivity for all remaining contrasts (including the other scan of the same contrast). For example, we correlated body selectivity (scan 1) with body selectivity (scan 2) to get a measure of within-category correlations within the ROI. We also correlated body selectivity with, for example, motion selectivity to test the relationship between different kinds of selectivity within this ROI. This results in a $4 \times 4$ matrix of correlations (reflecting the four contrasts tested) for each subject $\times$ ROI combination. The correlations were then Fisher transformed $\left[0.5 \times \log _{\mathrm{e}}((1+\mathrm{r}) /(1-\mathrm{r}))\right]$ before statistical testing.

\section{Results}

\section{Whole-brain group-average analyses}

Whole-brain group-average contrasts of moving versus static rings, intact versus scrambled objects, bodies versus chairs, and body parts versus object parts all produced substantially overlapping activation in the LOTC (Fig. 2). Spatial coordinates (Talairach and Tournoux, 1988) of the most-selective voxels of these regions are given in Table 1.

\section{Individually defined ROIs}

In all subjects, we were able to define, in both hemispheres, hMT, LO, wEBA, and pEBA. Mean coordinates (Talairach and Tournoux, 1988) of the peak voxel of these regions, averaged across subjects, are given in Table 2 . The mean activation peaks of these regions fell at most 5,8 , and $7 \mathrm{~mm}$ apart in the $x, y$, and $\mathrm{z}$ axes, respectively. The overlap among regions is illustrated in Figure 3 for six subjects. In the left hemisphere, there were marginally significant differences in mean $x$ and $y$ peak coordinates across the four ROIs $\left(F_{(1,15)}=2.2, p=0.11\right.$, and $F_{(1,15)}=2.5, p=0.076$, respectively), and the $z$ coordinates differed significantly $\left(F_{(1,15)}=\right.$ 4.6, $p=0.007)$. In the right hemisphere, there were significant 


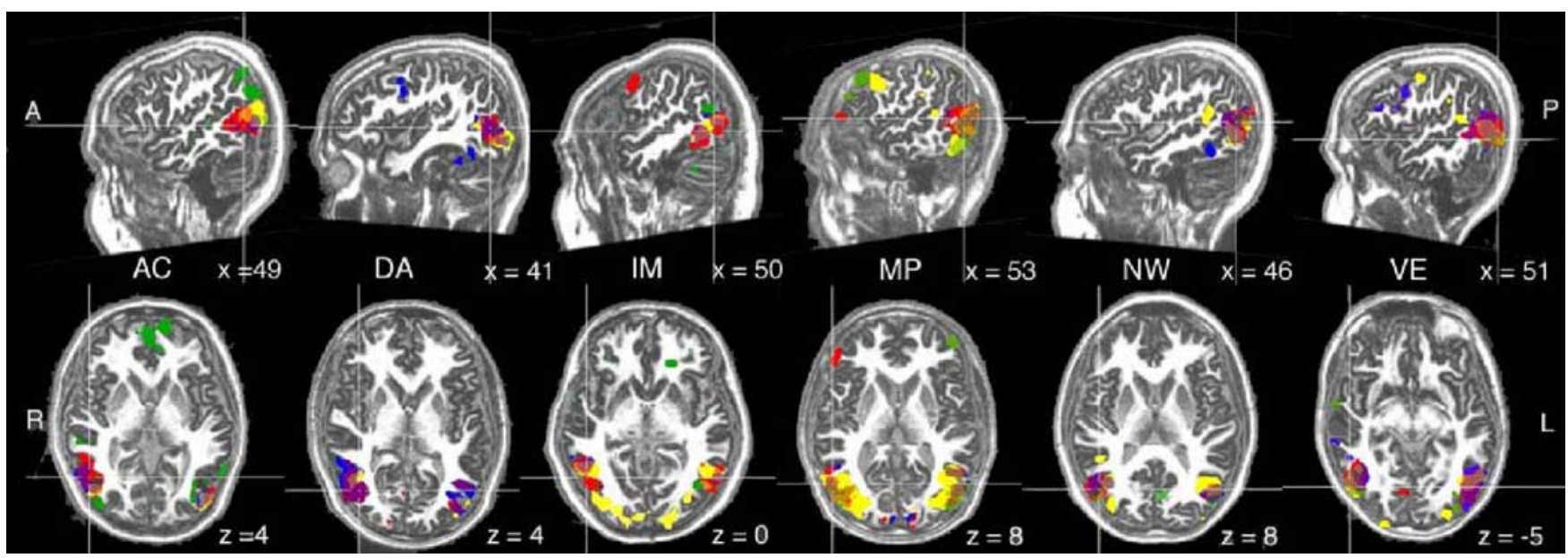

Figure 3. Results of experimental contrasts in six subjects, at a threshold of $p<0.0001$, uncorrected. Color conventions are as in Figure 2 . Slice coordinates, in Talairach space, are given to the bottom right of each image. A, Anterior; $P$, posterior; $R$, right; L, left.



Figure 4. Mean amplitude of response for each ROI to each contrast of interest. Each ROI for each subject was defined as the single lateral occipitotemporal voxel showing the greatest selectivity for the contrast in question. Data are given separately for left and right hemisphere. Data from the main diagonal (e.g., for motion selectivity in MT) are derived from split-half analyses so that voxel selection and response selectivity are determined with independent data sets. Error bars indicate SEM.

differences among the four regions on $x, y$, and $z$ coordinates $\left(F_{(1,15)}=4.9,6.7\right.$, and $5.7 ; p=0.005,0.001$, and 0.002 , respectively). A follow-up analysis tested whether distinct activation peaks would be revealed by the two different defining contrasts for EBA (bodies vs chairs and body parts vs object parts). These two peaks differed significantly in the $z$ coordinate of the left hemisphere $\left(F_{(1,15)}=4.6 ; p=0.05\right)$ and in the $y$ coordinate of the right hemisphere $\left(F_{(1,15)}=5.8 ; p<0.05\right)$.

Selectivity for each contrast was analyzed across ROIs (hMT, LO, wEBA, and pEBA) and hemispheres (left and right) in a series of 2 (condition) $\times 3$ (ROI) $\times 2$ (hemisphere) within-subjects ANOVAs (Fig. 4). Each ANOVA tested the selectivity for one contrast across ROIs and hemispheres. ROIs corresponding to the contrast tested were not included in the ANOVAs. For example, the ANOVA on object selectivity included hMT, wEBA, pEBA, but not LO, which is object selective by definition. [For comparison with other values in Fig. 4, within-region selectivity (e.g., for objects vs scrambled objects in $\mathrm{LO}$ ) was computed with split-half analyses, in which data from one scan defined the ROI and the other scan provided betas, and vice versa.] Because of the 
high number of tests involved, we set $\alpha=0.01$. This analysis revealed the extent to which motion, object, body, and body-part selectivity are found across the four ROIs examined. Note that ROIs for these analyses consisted only of the single most-selective voxel of each region.

For motion selectivity, we observed a significant main effect of hemisphere $\left(F_{(1,15)}=15.0 ; p<0.005\right)$, with overall larger responses in the right hemisphere, and a main effect of condition $\left(F_{(1,15)}=57.5 ; p<0.001\right)$, with overall larger responses to moving than static rings. These were modulated by a marginally significant interaction of hemisphere and condition $\left(F_{(1,15)}=7.9\right.$; $p=0.013)$. Motion selectivity was greater in the right hemisphere (mean difference of $\beta=3.7$ ) than in the left hemisphere (1.8). All effects involving ROI were nonsignificant (all $F<2.5$; all $p>$ 0.10 ), indicating motion selectivity did not vary significantly across LO, wEBA, and pEBA.

For object-form selectivity, the main effect of condition (intact vs scrambled) was significant $\left(F_{(1,15)}=37.5 ; p<0.001\right)$, and the main effect of ROI was marginally significant $\left(F_{(2,30)}=4.9\right.$; $p=0.014)$. However, these two factors significantly interacted $\left(F_{(2,30)}=17.6 ; p<0.001\right)$. Object selectivity was higher in the two EBA ROIs (mean difference: wEBA, 2.0; pEBA, 2.2) than in hMT (0.8), although even in hMT object selectivity was still statistically significant $\left(F_{(1,15)}=9.1 ; p<0.01\right)$.

For body-part selectivity, main effects of ROI and condition were significant $\left(F_{(2,30)}=7.4, p<0.005\right.$, and $F_{(1,15)}=122.1, p<$ 0.001 , respectively). These variables interacted significantly $\left(F_{(2,30)}=13.5 ; p<0.001\right)$. Selectivity for body parts, relative to object parts, was greater in wEBA (mean difference, 4.9) than in hMT (2.6) and LO (3.0). Body-part selectivity was significant in all three ROIs individually (all $F_{(1,15)}>43.2$; all $p<0.001$ ).

For whole-body selectivity, there was a significant main effect of hemisphere $\left(F_{(1,15)}=13.1 ; p<0.005\right)$, with stronger responses in the right hemisphere. A significant main effect of condition indicated overall higher responses to bodies than to chairs $\left(F_{(1,15)}=\right.$ 108.6; $p<0.001)$. Condition significantly interacted with ROI $\left(F_{(2,30)}=24.1 ; p<0.001\right)$. Body selectivity, relative to chairs, was greater in pEBA (mean difference, 5.6) than in hMT (3.1) and LO (2.5). Body selectivity was significant in all three ROIs individually $\left(\right.$ all $F_{(1,15)}>35.6$; all $\left.p<0.001\right)$.

To further confirm the selectivities of each ROI, selectivity for each pair of conditions was measured with a $t$ test, separately for each hemisphere and for each ROI (Fig. 4). With one exception, all ROIs showed significantly greater responses to moving versus static rings, to intact versus scrambled objects, to bodies versus chairs, and to body parts versus object parts (all $p<0.01$ ). The exception was that right hMT was not significantly selective for intact compared with scrambled objects $\left(t_{(15)}=1.4 ; p=0.18\right)$. In summary, the peak voxels of hMT, LO, wEBA, and pEBA all show significant selectivity for whole bodies, body parts, visual motion, and (with the exception of right hMT) intact objects.

\section{Pattern analyses}

\section{Combined ROIs}

Pattern analyses were applied to a general region of the LOTC, defined in each subject by the contrast of (motion + intact objects + bodies + body parts $)-$ (static rings + scrambled objects + chairs + object parts), at an uncorrected threshold of $p<$ 0.00001 . The volume of this region was, on average (SE), as follows: left hemisphere, $6.1 \mathrm{~cm}^{3}(0.6)$; right hemisphere, $9.2 \mathrm{~cm}^{3}$ (0.4). Within this region, the voxelwise correlations between patterns of selectivity produced by each contrast were calculated, separately for each subject and averaged across subjects (Table 3 ).
Table 3. Mean correlations (with SEs) between patterns of selectivity in lateral occipitotemporal cortex for different contrasts

\begin{tabular}{llllr}
\hline & Bodies & Body parts & Objects & \multicolumn{1}{c}{ Motion } \\
\hline Bodies & $0.60(0.04)$ & $0.52(0.04)$ & $0.00(0.05)$ & $0.00(0.03)$ \\
Body parts & & $0.69(0.04)$ & $0.11(0.05)$ & $-0.13(0.05)$ \\
Objects & & & $0.64(0.04)$ & $-0.15(0.04)$ \\
Motion & & & & $0.78(0.02)$
\end{tabular}

The region analyzed was that activated by contrasting (bodies, body parts, intact objects, moving rings) versus (chairs, object parts, scrambled objects, static rings), defined at $p<0.00001$ (uncorrected) in each subject individually $(n=16)$. Data were collapsed across hemispheres.

An initial ANOVA was conducted with hemisphere and correlation (10 unique pairwise correlations between selectivity patterns: 4 within-category and 6 between-category correlations) as factors. This analysis revealed a significant main effect of hemisphere $\left(F_{(1,15)}=5.0 ; p<0.05\right)$ and a significant main effect of correlation $\left(F_{(9,135)}=91.6 ; p<0.001\right)$, but no interaction between these factors $\left(F_{(9,135)}=0.5 ; \mathrm{NS}\right)$. This indicates that there was significant variation in the degree to which selectivity patterns were intercorrelated, but this did not depend on hemisphere, and additional analyses collapsed across this variable.

Strong, significant (all $t_{(15)}>12.3$; all $\left.p<0.001\right)$ correlations were found for activity elicited by the same contrasts across independent data sets (main diagonal in Table 3). This illustrates that each contrast produces a stable pattern of activity in the LOTC within subjects, across scans (cf. Haxby et al., 2001).

Analyses of off-diagonal correlations revealed a strong correlation between body selectivity [i.e., the response pattern to (bodies - chairs)] and body-part selectivity [i.e., the response pattern to (body parts - object parts) ]; $\left.r=0.52 ; t_{(15)}=12.3 ; p<0.001\right]$. This was similar to, but significantly lower than, the correlation between patterns elicited by repeated presentations of the same whole bodies $\left(r=0.60 ; t_{(15)}=2.8 ; p<0.05\right)$ and repeated presentations of body parts $\left(r=0.69 ; t_{(15)}=5.8 ; p<0.001\right)$.

Of the remaining off-diagonal correlations, there was a weak but significant positive correlation between body-part selectivity and object-form selectivity $\left(r=0.11 ; t_{(15)}=2.2 ; p<0.05\right)$. No other correlations were significantly positive. There were significant (weak) negative correlations between motion selectivity and both body-part selectivity $\left(r=-0.13 ; t_{(15)}=2.8 ; p<0.05\right)$ and object-form selectivity $\left(r=-0.15 ; t_{(15)}=3.5 ; p<0.005\right)$. The remaining off-diagonal correlations were nonsignificant $\left(t_{(15)} \ll 1\right)$.

Additional analyses tested, for each contrast, whether the within-contrast pattern correlation was significantly greater than the mean of all between-category correlations involving that contrast. That is, we asked for each correlation on the main diagonal (e.g., correlation of motion selectivity across separate scans), whether it was significantly greater than the off-diagonal correlations involving that contrast (e.g., the correlation between motion selectivity in one run and body selectivity in the other run). For each contrast, the within-category correlation was significantly greater than the mean of the between-category correlations involving that contrast ( all $t_{(15)}>9.9 ; p<0.001$ ). Additionally, a statistically significant difference was found in every pairwise comparison of a within-category pattern versus each between-category pattern involving that contrast [e.g., motion motion vs motion - body parts (all $\left.t_{(15)}>5.8 ; p<0.001\right)$, except bodies - bodies vs bodies - body parts $\left.\left(t_{(15)}=2.8 ; p<0.05\right)\right]$.

We conducted additional multi-voxel analyses of combined ROIs defined (again in each individual subject) with different statistical thresholds. Whether ROIs were defined with false discovery rate thresholds of $\mathrm{q}<0.01$ or $\mathrm{q}<0.001$, or a standard uncorrected $p$ value of $p<0.000001$, highly similar results were 
Table 4. Mean correlations (with SEs) between patterns of selectivity in lateral occipitotemporal cortex for different contrasts

\begin{tabular}{llllr}
\hline & Bodies & Body parts & Objects & \multicolumn{1}{c}{ Motion } \\
\hline Bodies & $0.67(0.04)$ & $0.54(0.03)$ & $0.14(0.05)$ & $0.03(0.03)$ \\
Body parts & & $0.75(0.03)$ & $0.25(0.05)$ & $-0.07(0.03)$ \\
Objects & & & $0.71(0.03)$ & $-0.18(0.03)$ \\
Motion & & & & $0.76(0.02)$
\end{tabular}

The region analyzed was that activated by identifying the union of $h M T, L 0$, and EBA in each subject individually $(n=16)$, defined at $p<0.0001$ (uncorrected) in each subject individually. Data were collapsed across hemispheres. All correlations were determined on the basis of voxelwise patterns of activity from independent data sets.

obtained. These results are provided in supplemental Tables S1-S3 (available at www.jneurosci.org as supplemental material).

\section{Union ROIs}

Pattern analyses were also performed on data defined by the union of individually defined hMT, LO, and EBA ROIs (Table 4). That is, these voxels were significantly selective for motion, object form, or body form. The volume of this region was, on average (SE), as follows: left hemisphere, $11.6 \mathrm{~cm}^{3}$ (1.5); right hemisphere, $15.3 \mathrm{~cm}^{3}$ (1.6). Within-category pattern correlations were all significantly different from zero (all $t_{(15)}>13.7 ; p<$ $0.001)$. Again, the correlation between body selectivity and bodypart selectivity was high $(r=0.54)$ and differed from zero $\left(t_{(15)}=\right.$ $14.2 ; p<0.001)$.

Other between-category correlations that were weak but significantly greater than zero in this analysis were between body selectivity and object selectivity $\left(r=0.14 ; t_{(15)}=2.9 ; p<0.05\right)$ and between body-part selectivity and object selectivity $(r=0.25$; $\left.t_{(15)}=4.4 ; p<0.005\right)$.

For each contrast, the within-category correlation was significantly greater than the mean of the between-category correlations involving that contrast (all $t_{(15)}>10.1 ; p<0.001$ ). Additionally, a statistically significant difference was found in every pairwise comparison of a within-category pattern versus each between-category pattern involving that contrast $\left(\right.$ all $t_{(15)}>4.6$; $p<0.001)$.

\section{Intersection ROIs}

Here we tested whether the region at the intersection of hMT, LO, and EBA would show a similar pattern of correlations as the previous analyses. We reasoned that within this intersection region, there might be a subpopulation of neurons with a common functional role in responding to motion, objects, bodies, and body parts. In 14 of 16 subjects, we identified a set of voxels in both hemispheres in the intersection of hMT, LO, and EBA (union of wEBA and pEBA). That is, these voxels were significantly selective for motion, object form, and body form. The volume of this region was, on average (SE), as follows: left hemisphere, $0.6 \mathrm{~cm}^{3}$ (0.14); right hemisphere, $0.9 \mathrm{~cm}^{3}(0.21)$. Note that the substantially smaller ROIs used in these analyses result from taking the intersection of ROIs and will result in noisier activation patterns. Analyses were collapsed across hemisphere because this factor did not interact with the pattern of correlations.

The results of this analysis are given in Table 5 . As in the previous analyses, all within-category correlations were significantly greater than zero (all $t_{(13)}>2.5$; all $p<0.001$, except objects at $p<0.05$ ), as was the correlation between body and body-part selectivity $\left(r=0.39 ; t_{(13)}=5.1 ; p<0.001\right)$. Of the remaining off-diagonal correlations, only the correlation between body selectivity and motion selectivity approached significance (albeit weak; $r=0.16 ; t_{(13)}=2.8 ; p<0.05$ ).

For each contrast, the within-category correlation was signif-
Table 5. Mean correlations (with SEs) between patterns of selectivity in lateral occipitotemporal cortex for different contrasts

\begin{tabular}{lllrr}
\hline & Bodies & Body parts & \multicolumn{1}{c}{ Objects } & \multicolumn{1}{c}{ Motion } \\
\hline Bodies & $0.53(0.08)$ & $0.39(0.07)$ & $-0.06(0.05)$ & $0.16(0.06)$ \\
Body parts & & $0.60(0.09)$ & $0.07(0.03)$ & $0.07(0.07)$ \\
Objects & & & $0.23(0.09)$ & $-0.03(0.05)$ \\
Motion & & & & $0.38(0.08)$
\end{tabular}

The region analyzed was that activated by identifying the intersection of $\mathrm{hMT}, \mathrm{LO}$, and EBA in each subject individually $(n=14)$, defined at $p<0.0001$ (uncorrected) in each subject individually. Data were collapsed across hemispheres. All correlations were determined on the basis of voxelwise patterns of activity from independent data sets.

icantly greater than the mean of the between-category correlations involving that contrast: motion: $t_{(13)}=3.4, p<0.005$; objects: $t_{(13)}=2.5, p<0.05$; bodies: $t_{(13)}=5.6, p<0.001$; body parts: $t_{(13)}=5.0, p<0.001$. Additionally, a statistically significant difference was found in every pairwise comparison of a withincategory pattern versus each between-category pattern involving that contrast [all $t_{(15)}>2.4 ; p<0.05$, except (objects - objects vs body parts - objects); $t_{(15)}=1.6 ; p=0.12$ ].

Thus, even in the intersection of hMT, LO, and EBA, where each voxel shows highly significant selectivity to motion, object structure, and bodies and body parts, the patterns of selectivity elicited by these stimuli remain essentially distinct.

\section{Discussion}

We found that the lateral occipitotemporal activations elicited by motion, object form, bodies, and body parts overlap substantially at the group-average level. When hMT, LO, wEBA, and pEBA were defined functionally within individual subjects, they overlapped, and their peak voxels were, on average, highly similar to each other, although not identical. Strikingly, we found that every ROI in each hemisphere showed significant selectivity for motion, intact objects, bodies, and body parts (with the exception of intact objects in right hMT). This is particularly remarkable given that for this analysis, each ROI was defined on the basis of the single most selective voxel in each subject (in spatially unsmoothed data).

Multi-voxel analyses, however, revealed that there are three relatively independent patterns of $\mathrm{AMRI}$ activity in LOTC elicited by visual motion, by object form, and by the form of the human body, each compared with a matched control. What is the implication of this finding for the organization of LOTC? At the level of single neurons, a case can be made that multi-voxel analysis of fMRI data allows for neural organization to be examined at subvoxel resolution. In V1, for example, consideration of activity patterns allows the discrimination of viewed grating orientations, presumably because of varying concentrations of different orientation columns across different voxels (Kamitani and Tong, 2005). Thus, multi-voxel fMRI analyses can confirm and indeed take advantage of known organization at the single-unit level. However, where the neuronal organization is a priori less clear (as in human extrastriate cortex), the pattern data are consistent with a range of situations. Specifically, the present data could indicate the following: (1) separate but interwoven populations of neurons, each highly selective for motion, object form, or body part form; (2) a single population of neurons, the members of which respond in varying (but statistically independent) degrees to motion, objects, and bodies/body parts; or (3) an intermediate scenario with characteristics of (1) and (2).

What can be ruled out, however, is that (even in the region of overlap between fMRI activations) the same pattern of neural activity is elicited across this region by the stimuli tested here. We 
take this to indicate that (in contrast to the standard fMRI overlap logic) the processing of visual motion, object form, and body form in LOTC are functionally independent. That is, the computations involved in analyzing these stimuli are distinct. Of course, the fact that the neural instantiation of these computations lies in close proximity may be significant. For example, the region as a whole may integrate motion, object, and body-form cues to interpret dynamic human-object interactions.

The pattern of results for bodies and body parts indicates something of the nature of the representation of the human figure in this region. Previous work on the EBA has shown a greater response in this region to whole bodies than to body parts (Downing et al., 2001). However, this finding is ambiguous: the difference could arise because EBA preferentially processes the holistic form of the body, or instead favors body parts, but responds more to whole bodies because of the greater number of such parts in each image. The present finding of highly similar patterns of activity elicited by whole bodies and by body parts is more supportive of the latter account. Bodies and body parts apparently engage similar activity across the population of neurons in LOTC, although at a global level, the stimuli differ greatly in appearance. If the body representation of these neurons was primarily selective for the appearance of the entire body, we would have expected whole-body stimuli to evoke a unique pattern of activity that would have been matched, to a lesser extent, by body parts.

The present findings have general methodological implications for the interpretation of within-subject fROI studies. Specifically, many studies have used the fROI approach to identify a region and measure its response properties, by rough analogy to single-unit neurophysiology (Saxe et al., 2006; see also Friston et al., 2006). However, our results indicate that the finding that a functionally defined region (or even the single most selective voxel of a region) significantly discriminates among a different set of stimuli may not be indicative of the functional role of that region in processing those stimuli (see also Peelen and Downing, 2005a; Peelen et al., 2006).

From a practical perspective, then, investigations of an fROI must take into account the presence of other nearby or overlapping selective populations. For example, our results suggest that the finding that area hMT (as localized with the typical fROI method) responds selectively to bodies (Spiridon et al., 2005) may be reinterpreted as a response of a body-specific neural mechanism that is captured in at least some of the voxels identified as hMT. Future investigations of the visual cortex, or indeed other brain areas where multiple independent populations may be suspected to overlap, would benefit from concurrent withinsubject measurement of multiple kinds of selectivity.

For example, consider an fROI study that (1) assumes a population of human body-selective neurons exists in LOTC, and (2) aims to measure the response of this population to visual depictions of animals, relative to inanimate control stimuli (cf. Downing et al., 2001). Whole-brain and standard fROI approaches would not be able to disentangle body selectivity in this region from the motion and object-form selectivity attributed to hMT and LO. A more powerful analysis would measure animal selectivity on a voxelwise basis across this region and demonstrate that this correlates positively across voxels with human body selectivity but does not relate to motion selectivity nor to object-form selectivity. This approach may prove still more valuable as fMRI studies are performed at increasingly high resolution. At high resolutions, areas that appear unitary at typical resolutions (and therefore measurable as a single entity) may begin to fragment
(Grill-Spector et al., 2006). At increased spatial resolutions, then, defining, for example, an "EBA" may be less practical than measuring variations in body selectivity across a broad region and relating this selectivity on a voxel-by-voxel basis to other variables of interest. In this way, in which several types of selectivity exist at close quarters (perhaps indicating multiple independent neural populations), one of these can be isolated for investigation without contamination from the others.

How does the approach developed here relate to multi-voxel methods applied in other recent studies (Haynes and Rees, 2005; Kamitani and Tong, 2005, 2006; Polyn et al., 2005; Kriegeskorte et al., 2006)? Although our approach shares in common with these studies the consideration of multi-voxel patterns of fMRI activity, our aim is essentially different from that of these researchers. Most applications of multi-voxel fMRI analysis have been aimed at "brain reading," attempting to determine which of several cognitive states a subject was in at a given point in time, based on the pattern of blood oxygenation level-dependent activity either in an ROI or searching across the entire brain for regions that convey this information. In contrast, our aim is closer to that of Haxby et al. (2001), in that we seek to uncover evidence on the functional organization of cortex that falls within overlapping fMRI activations. Here we focus on LOTC, but our approach will be applicable to other brain regions.

The limitations to the approach developed here are shared, to some extent, with other multi-voxel techniques used in recent studies (Haynes and Rees, 2006; Norman et al., 2006). First, the spatial resolution of the data imposes a fundamental limit; multivoxel methods rely on stable spatial variations in selectivity that must be detectable at the sampled resolution. Second, although here we used correlations as a measure of the similarity of spatial activity patterns, other approaches have been developed that may detect higher-order similarities (Cox and Savoy, 2003) (but see Kamitani and Tong, 2005). Finally, the interpretation of pattern analyses depends on the specific stimuli used that limit the generality of inferences about the function of a given neural network. For example, claims that a region carries information about the human body form in a distributed pattern of neural activity are stronger to the extent that a variety of body (and non-body) stimuli are tested concurrently.

To conclude, our results show how recently developed multivoxel analysis approaches may be adapted to aid in understanding the functional significance of overlapping fMRI activations in extrastriate cortex and, by extension, anywhere in the brain. This issue is relevant for many areas of cognitive neuroscience.

\section{References}

Boynton GM, Engel SA, Glover GH, Heeger DJ (1996) Linear systems analysis of functional magnetic resonance imaging in human V1. J Neurosci 16:4207-4221.

Cox DD, Savoy RL (2003) Functional magnetic resonance imaging (fMRI) "brain reading": detecting and classifying distributed patterns of fMRI activity in human visual cortex. NeuroImage 19:261-270.

Downing PE, Jiang Y, Shuman M, Kanwisher N (2001) A cortical area selective for visual processing of the human body. Science 293:2470-2473.

Dumoulin SO, Bittar RG, Kabani NJ, Baker CL, Le Goualher G, Bruce Pike G, Evans AC (2000) A new anatomical landmark for reliable identification of human area V5/MT: a quantitative analysis of sulcal patterning. Cereb Cortex 10:454-463.

Friston KJ, Rotshtein P, Geng JJ, Sterzer P, Henson RN (2006) A critique of functional localisers. NeuroImage 30:1077-1087.

Grill-Spector K, Kushnir T, Edelman S, Avidan G, Itzchak Y, Malach R (1999) Differential processing of objects under various viewing conditions in the human lateral occipital complex. Neuron 24:187-203.

Grill-Spector K, Sayres R, Ress D (2006) High-resolution imaging reveals 
highly selective nonface clusters in the fusiform face area. Nat Neurosci 9:1177-1185.

Haxby JV, Gobbini MI, Furey ML, Ishai A, Schouten JL, Pietrini P (2001) Distributed and overlapping representations of faces and objects in ventral temporal cortex. Science 293:2425-2430.

Haynes JD, Rees G (2005) Predicting the orientation of invisible stimuli from activity in human primary visual cortex. Nat Neurosci 8:686-691.

Haynes JD, Rees G (2006) Decoding mental states from brain activity in humans. Nat Rev Neurosci 7:523-534.

Iacoboni M, Woods RP, Brass M, Bekkering H, Mazziotta JC, Rizzolatti G (1999) Cortical mechanisms of human imitation. Science 286:2526-2528.

Jiang Y, Kanwisher N (2003) Common neural substrates for response selection across modalities and mapping paradigms. J Cogn Neurosci 15:1080-1094.

Kamitani Y, Tong F (2005) Decoding the visual and subjective contents of the human brain. Nat Neurosci 8:679-685.

Kamitani Y, Tong F (2006) Decoding seen and attended motion directions from activity in the human visual cortex. Curr Biol 16:1096-1102.

Kourtzi Z, Bülthoff HH, Erb M, Grodd W (2002) Object-selective responses in the human motion area MT/MST. Nat Neurosci 5:17-18.

Kourtzi Z, Erb M, Grodd W, Bülthoff HH (2003) Representation of the perceived 3-D object shape in the human lateral occipital complex. Cereb Cortex 13:911-920.

Kriegeskorte N, Goebel R, Bandettini P (2006) Information-based functional brain mapping. Proc Natl Acad Sci USA 103:3863-3868.

Malach R, Reppas JB, Benson RR, Kwong KK, Jiang H, Kennedy WA, Ledden PJ, Brady TJ, Rosen BR, Tootell RB (1995) Object-related activity revealed by functional magnetic resonance imaging in human occipital cortex. Proc Natl Acad Sci USA 92:8135-8139.

Marois R, Chun MM, Gore JC (2004) A common parieto-frontal network is recruited under both low visibility and high perceptual interference conditions. J Neurophysiol 92:2985-2992.
Norman KA, Polyn SM, Detre GJ, Haxby JV (2006a) Beyond mind-reading: multi-voxel pattern analysis of fMRI data. Trends Cogn Sci 10:424-430.

Peelen MV, Downing PE (2005a) Is the extrastriate body area involved in motor actions? Nat Neurosci 8:125.

Peelen MV, Downing PE (2005b) Within-subject reproducibility of category-specific visual activation with functional MRI. Hum Brain Mapp 25:402-408.

Peelen MV, Wiggett AJ, Downing PE (2006) Patterns of fMRI activity dissociate overlapping functional brain areas that respond to biological motion. Neuron 49:815-822.

Polyn SM, Natu VS, Cohen JD, Norman KA (2005) Category-specific cortical activity precedes retrieval during memory search. Science 310:1963-1966.

Price CJ, Friston KJ (1997) Cognitive conjunction: a new approach to brain activation experiments. NeuroImage 5:261-270.

Saxe R, Brett M, Kanwisher N (2006) Divide and conquer: a defense of functional localizers. NeuroImage 30:1088-1096; discussion 1097-1099.

Singer T, Seymour B, O'Doherty J, Kaube H, Dolan RJ, Frith CD (2004) Empathy for pain involves the affective but not sensory components of pain. Science 303:1157-1162.

Spiridon M, Fischl B, Kanwisher N (2005) Location and spatial profile of category-specific regions in human extrastriate cortex. Hum Brain Mapp 27:77-89.

Talairach J, Tournoux P (1988) Co-planar stereotaxic atlas of the human brain. Stuttgart, Germany: Thieme.

Tootell RB, Reppas JB, Kwong KK, Malach R, Born RT, Brady TJ, Rosen BR, Belliveau JW (1995) Functional analysis of human MT and related visual cortical areas using magnetic resonance imaging. J Neurosci 15:3215-3230.

Wojciulik E, Kanwisher N (1999) The generality of parietal involvement in visual attention. Neuron 23:747-764. 